\title{
Electrically controlled nuclear polarization of individual atoms
}

Kai Yang1, Philip Willke ${ }^{1,2,3}$, Yujeong Bae ${ }^{1,2,3}$, Alejandro Ferrón4, Jose L. Lado ${ }^{5,6}$, Arzhang Ardavan7, Joaquín Fernández-Rossier5,8, Andreas J. Heinrich2,3,, , and Christopher P. Lutz1,*

1IBM Almaden Research Center, San Jose, CA 95120, USA

${ }^{2}$ Center for Quantum Nanoscience, Institute for Basic Science (IBS), Seoul 03760, Republic of Korea

3Department of Physics, Ewha Womans University, Seoul 03760, Republic of Korea

4Instituto de Modelado e Innovación Tecnológica (CONICET-UNNE), and Facultad de Ciencias Exactas, Naturales y Agrimensura, Universidad Nacional del Nordeste, Avenida Libertad 5400, W3404AAS Corrientes, Argentina

5QuantaLab, International Iberian Nanotechnology Laboratory (INL), Avenida Mestre José Veiga, 4715-310 Braga, Portugal

6Institute for Theoretical Physics, ETH Zurich, 8093 Zurich, Switzerland

${ }^{7}$ Clarendon Laboratory, Department of Physics, University of Oxford, Oxford OX1 3PU, UK.

8Departamento de Física Aplicada, Universidad de Alicante, San Vicente del Raspeig 03690, Spain

* Corresponding authors: A.J.H. (heinrich.andreas@qns.science) and C.P.L. (cplutz@us.ibm.com)

Nuclear spins are sensitive probes in analytical chemistry ${ }^{1,2}$ and materials science ${ }^{3,4}$ as well as promising candidates for quantum information processing ${ }^{5-9}$. Manipulating nuclear spins in condensed matter systems ${ }^{5-7,10,11}$ is difficult due to the small nuclear magnetic moment, leading to low polarizations, and addressing them individually is particularly challenging 6,7,12. Here, we polarize nuclear spins of individual copper $(\mathrm{Cu})$ atoms on a surface using spinpolarized current in a scanning tunneling microscope. By employing the electron-nuclear flipflop hyperfine interaction, the spin angular momentum is transferred to the nucleus of individual $\mathrm{Cu}$ atoms. The direction and magnitude of the nuclear polarization is controlled by the direction and amplitude of the current. We drive resonant transitions between polarized nuclear spin states, which can be used to sense the local magnetic environment of the $\mathrm{Cu}$ electron spin. This nuclear spin-transfer torque effect should be also present in other electrically accessible nuclear spin systems ${ }^{6-8}$ such as the nuclear spins embedded in singlemolecule magnets 6 , and thus offers a general route towards electrically-controlled nuclear spin devices.

Nuclear magnetic resonance (NMR) is a powerful tool for probing local magnetic environments in condensed matter systems, ranging from magnetic ordering in high temperature superconductors ${ }^{13,14}$ and spin liquids ${ }^{15}$, to low-dimensional quantum magnetism in nanomagnets containing exchange-coupled ions ${ }^{16,17}$. Increasing the sensitivity and spatial resolution of NMR spectroscopy to the atomic scale requires strongly polarizing a single or a few nuclear spins, as well as driving and detecting them individually. However, the tiny magnetic moments of nuclear spins yield only weak thermal polarization. In 1953, Overhauser proposed that nuclear spin polarization exceeding the thermal equilibrium value, known as hyperpolarization, can be achieved through hyperfine coupling with electron spins ${ }^{4,18}$. The fundamental mechanism is the conservation of 
angular momentum: electron spin "flips" and nuclear spin "flops", so that angular momentum is transferred from the electron to the nucleus. Such hyperpolarization, first achieved by saturating electron spin resonance in metals using microwave fields ${ }^{4}$, enables applications such as in vivo magnetic resonance imaging using nanoparticles ${ }^{19}$. In the context of spin-based quantum information processing, ensemble ( $\sim 10^{5}$ spins) hyperpolarization using optical pumping has been widely used in quantum dots ${ }^{9}$ and ${ }^{31} \mathrm{P}$ doped silicon ${ }^{10,20,21}$.

Electrical control of nuclear spins can be achieved using alternating6,8 or static ${ }^{11}$ electric fields, as well as electric current 22,23 . Compared to magnetic ${ }^{7}$ and optical control ${ }^{12}$, electrical control is particularly appealing for applications, because electric fields are relatively easy to generate locally and allow individual spins to be addressed 6 . Here, we use electric current to control nuclear magnetism of individual atoms for the first time. We demonstrate all-electric polarization and resonant driving of the single nuclear spin of a $\mathrm{Cu}$ atom by using a scanning tunneling microscope (STM) (Fig. 1a). The Cu nuclear spin is polarized with either polarity, by as much as $30 \%$, which is $\sim 17$ times greater than thermal polarization at $1 \mathrm{~K}$. This is achieved by employing a spin-transfer torque effect at the single-atom level (Fig. 1b). The current-controlled nuclear polarization permits detection of NMR, which is then used to sense the magnetic environment of the Cu atom.
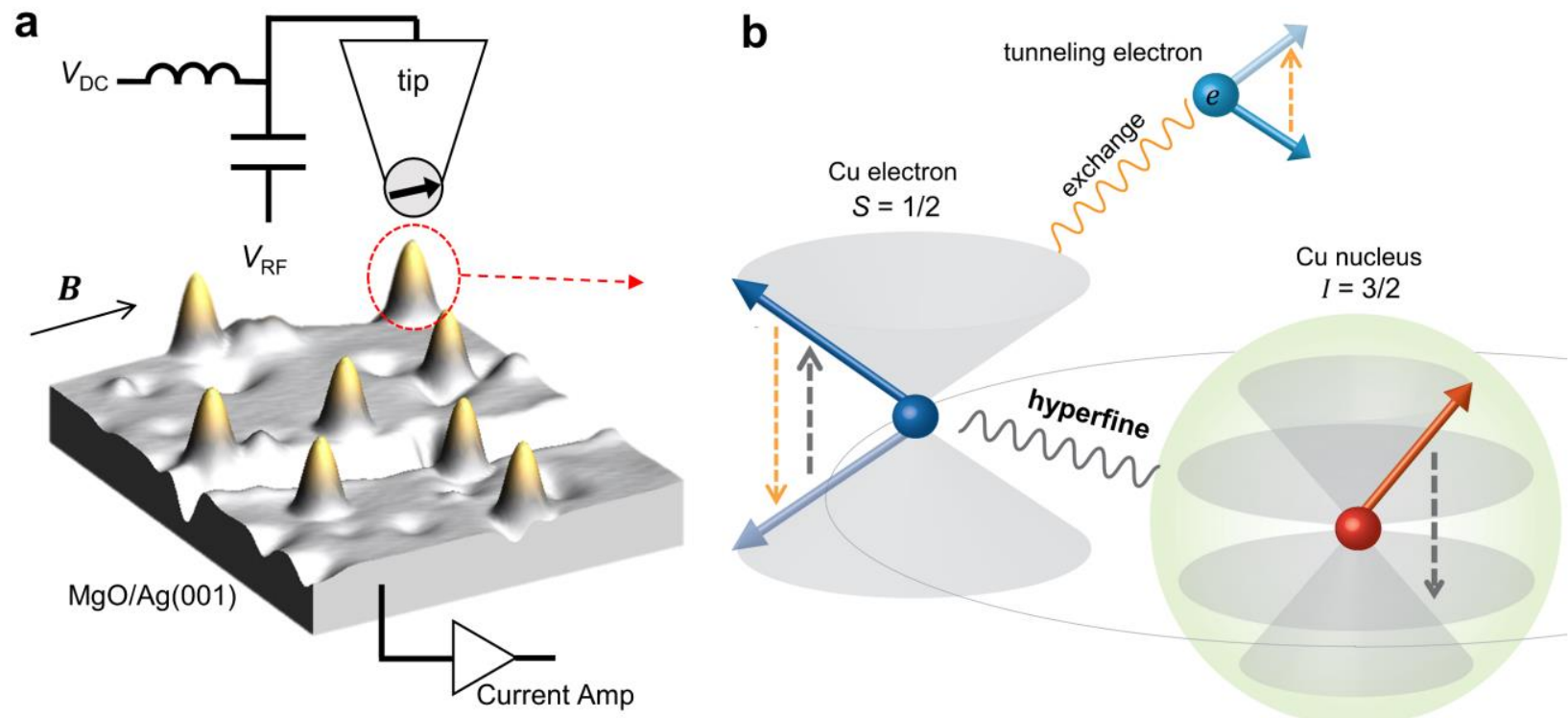

Figure 1 | Electrical polarization of the nuclear spin of a Cu atom on MgO. a, Schematic of the experimental set-up consisting of an STM with ESR capability, with an STM image of $\mathrm{Cu}$ atoms (yellow peaks) on bilayer MgO on $\mathrm{Ag}(001)$ (setpoint: $\left.V_{\mathrm{DC}}=50 \mathrm{mV}, I_{\mathrm{DC}}=20 \mathrm{pA}\right)$. Both $\mathrm{DC}\left(V_{\mathrm{DC}}\right)$ and radio-frequency voltages $\left(V_{\mathrm{RF}}\right)$ are applied to the STM junction. Arrow at tip apex indicates a magnetic atom to give spin-polarized tip. $\mathbf{b}$, Mechanism of the nuclear spin polarization for the coupled electron $(S=1 / 2)$ and nuclear $(I=3 / 2)$ spin system. The tunneling electron changes the orientation of the $\mathrm{Cu}$ electron spin by exchange interaction (dashed orange arrows), and angular momentum is subsequently transferred from the $\mathrm{Cu}$ electron to the $\mathrm{Cu}$ nucleus by the flip-flop hyperfine interaction (dashed grey arrows). 
The $\mathrm{Cu}$ atoms were deposited on a MgO decoupling layer grown on a $\mathrm{Ag}(001)$ substrate (Fig. 1a). This set-up makes individual $\mathrm{Cu}$ atoms electrically accessible to the probe tip of STM, by measuring the time-average current $\left(I_{\mathrm{DC}}\right)^{24}$. Each $\mathrm{Cu}$ atom adsorbs on top of the oxygen site (Fig. S1a), and has an electron spin $S=1 / 2$ as determined below. Cu occurs naturally in two stable isotopes ${ }^{25:}{ }^{63} \mathrm{Cu}$ $(\sim 69 \%)$ and ${ }^{65} \mathrm{Cu}(\sim 31 \%)$, and both have a nuclear spin $I=3 / 2$. We model the coupled electronnuclear system of $\mathrm{Cu}(S=1 / 2, I=3 / 2)$ in a magnetic field $\mathbf{B}$ by using an isotropic hyperfine coupling term and electron Zeeman term:

$$
H=A \mathbf{S} \cdot \mathbf{I}+\gamma_{e} \mathbf{B} \cdot \mathbf{S}
$$

Here $A$ is the hyperfine constant and $\gamma_{e}$ is the electron gyromagnetic ratio. The anisotropic components of the hyperfine coupling and electric quadrupole coupling are much weaker $(<100 \mathrm{MHz}$ according to density functional theory (DFT) calculations in Supplementary Sec. 2), and are omitted here, as is the nuclear Zeeman energy.

We probe the quantum states of individual $\mathrm{Cu}$ atom by using inelastic electron tunneling spectroscopy (IETS) ${ }^{26}$ and electron spin resonance (ESR) ${ }^{27-29}$. An in-plane magnetic field is applied to set the electron Zeeman energy of $\mathrm{Cu}$. IETS reveals spin excitations between states with electron spin quantum number $m_{\mathrm{S}}= \pm 1 / 2$ (labeled as $\uparrow$ and $\downarrow$ in the following), by measuring differential conductance $(\mathrm{d} I / \mathrm{d} V)$ spectra (Fig. 2a). By fitting the electron Zeeman splitting as a function of $B$ field (Fig. 2a, inset) ${ }^{30}$, we obtain an electron $g$ factor of $1.98 \pm 0.10$, and $\gamma_{e}=g \mu_{B} / h=27.71 \pm 1.40 \mathrm{GHz} / \mathrm{T}$ ( $h$ is the Planck's constant and $\mu_{\mathrm{B}}$ is the Bohr magneton). This suggests that Cu has an electron spin $S$ $=1 / 2$, as confirmed by our DFT calculations.

We resolve the hyperfine structure of individual $\mathrm{Cu}$ atom by ESR ${ }^{27,29}$, with an energy resolution of $\sim 100 \mathrm{neV}$. Using a spin-polarized tip (Fig. S3a), we drive and detect the ESR transitions between $\uparrow$ and $\downarrow$ states of $\mathrm{Cu}$. The four ESR peaks (Fig. 2b) correspond to the four different orientations of the nuclear spin $I=3 / 2$ (Fig. 2d). The hyperfine constant $A$ can be extracted from the four ESR frequencies: $A=\left(f_{\mathrm{II}}+f_{\mathrm{IV}}-f_{\mathrm{I}}-f_{\mathrm{III}}\right) / 2$ (Supplementary Sec. 6). The histogram of $A$ values for 31 individual $\mathrm{Cu}$ atoms (Fig. 2b, right inset) shows two separate Gaussian peaks at $2.86 \pm 0.03 \mathrm{GHz}$ and $3.05 \pm 0.07 \mathrm{GHz}$, corresponding to the two isotopes ${ }^{63} \mathrm{Cu}$ and ${ }^{65} \mathrm{Cu}$, respectively. The ratio of $0.93 \pm$ 0.02 between the two $A$ values agrees well with the ratio of 0.9336 measured by the atomic beam technique ${ }^{25}$. This demonstrates the capability of ESR-STM to distinguish different isotopes with atomic precision, even when the nuclear spin magnitude is equal.

The hyperfine constant is sensitive to the chemical environment of an atom ${ }^{29,31}$ and here the bonding configuration of $\mathrm{Cu}$ on MgO. DFT calculations revealed that the electron spin occupies mainly ( $\sim 60 \%)$ the $4 s$ orbital (Fig. $2 \mathrm{~b}$, left inset). The large hyperfine constant of $\mathrm{Cu}$ on $\mathrm{MgO}$, compared to 
in other environments ${ }^{31}$, thus arises from the large $s$-electron density at the nucleus, which yields an unusually large Fermi contact interaction ${ }^{4}$. The DFT calculated values for the two isotopes $(A=3.76$ $\mathrm{GHz}$ and $4.02 \mathrm{GHz}$ ) agree well with the experimental values.

a

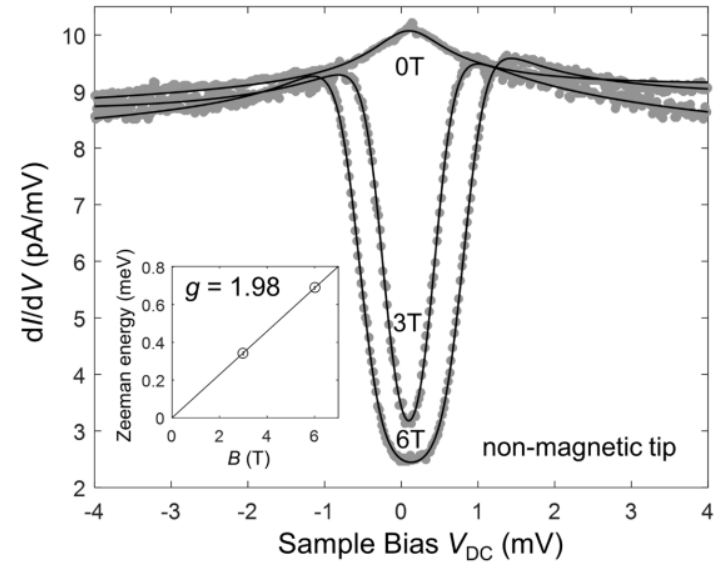

C 15

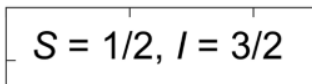

(1)

$|6\rangle$ b

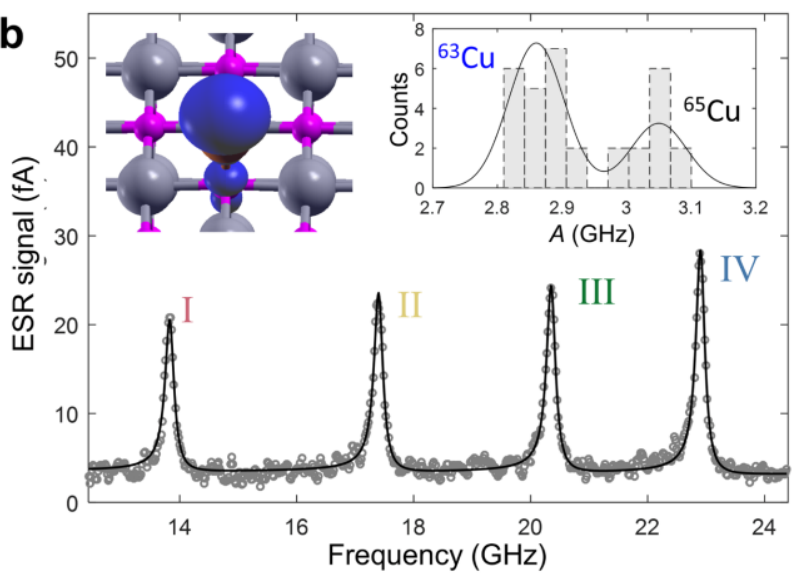

d

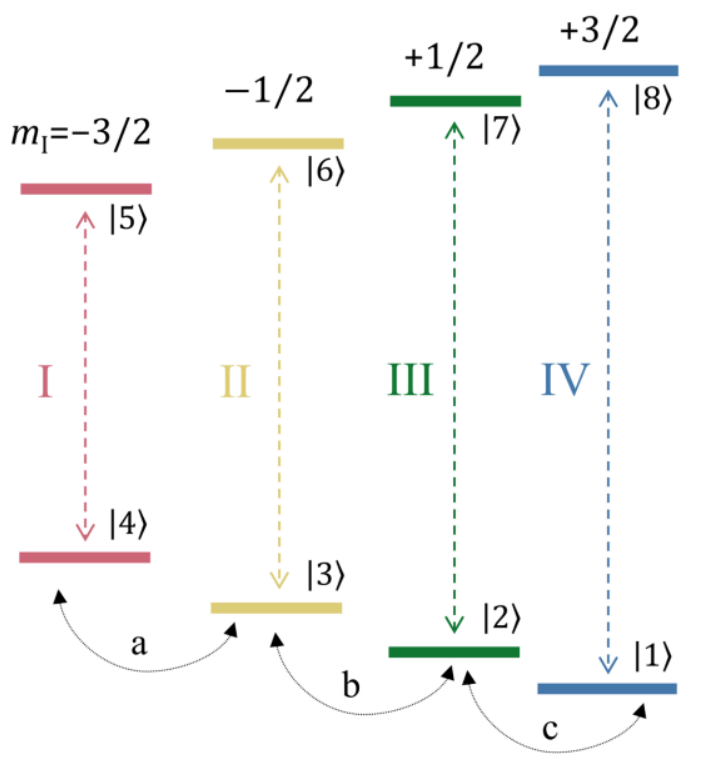

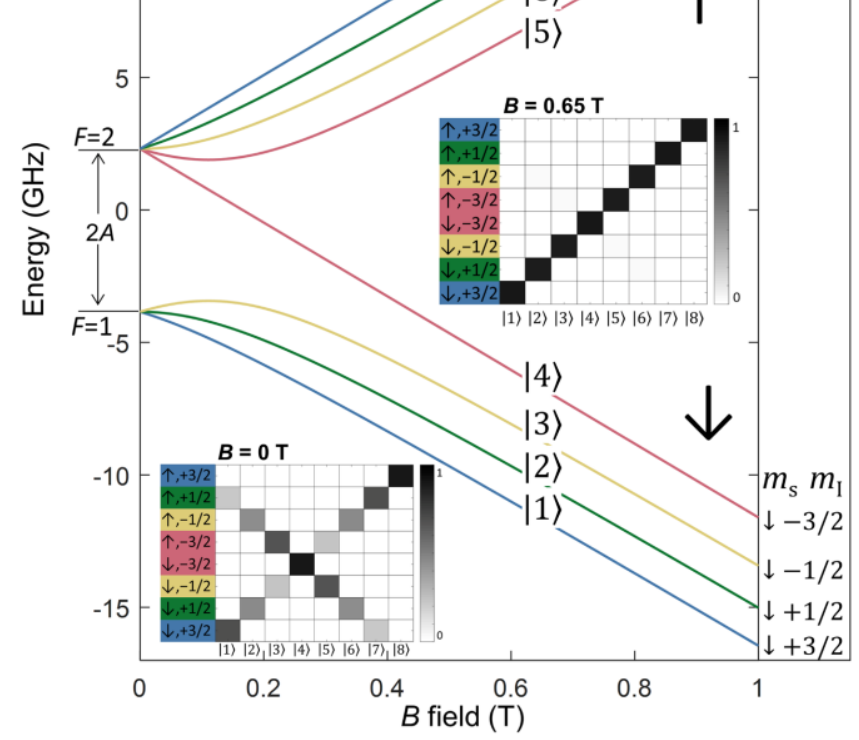

$\uparrow+1 / 2$

|8) $\begin{aligned} & \uparrow-1 / 2 \\ & \uparrow-3 / 2\end{aligned}$

$\uparrow$

Figure 2 | Electronic and hyperfine structures of a single $\mathrm{Cu}$ atom on $\mathrm{MgO}$. a, $\mathrm{d} I / \mathrm{d} V$ spectra of $\mathrm{Cu}$ at different magnetic fields with a non-magnetic tip (setpoint: $V_{\mathrm{DC}}=10 \mathrm{mV}, I_{\mathrm{DC}}=100 \mathrm{pA} ; T=0.6 \mathrm{~K}$ ). Spectra are vertically shifted for clarity. The position of the conductance steps reveals the Zeeman energy. The zero-bias peak at zero field is a Kondo resonance. Inset: magnetic field dependence of the Zeeman energy. Linear fit constrained to zero Zeeman energy at zero field yields a $g$ factor of $1.98 \pm 0.10$. b. ESR spectrum of a ${ }^{65} \mathrm{Cu}$ atom (setpoint: $V_{\mathrm{DC}}=-20 \mathrm{mV}, I_{\mathrm{DC}}=40 \mathrm{pA} ; V_{\mathrm{RF}}=15 \mathrm{mV}$, total field $B=0.65 \mathrm{~T}$ consisting of an external field of $0.765 \mathrm{~T}$ and an effective tip field, $T=1.2 \mathrm{~K}$ ). Inset: (Left) Calculated spin density (dark blue) of $\mathrm{Cu}$ on $\mathrm{MgO}$. (Right) Histogram of hyperfine constant $A$ of $31 \mathrm{Cu}$ atoms, fit by two Gaussians. c, Energy diagram of the electron- 
nuclear spin system as a function of $B$ field. Insets: Schematic of the energy eigenstates at $B=0$ and $0.65 \mathrm{~T}$. Each column represents one eigenstate. The grey level represents the probability amplitudes in the basis of Zeeman product states $\left|m_{\mathrm{S}}, m_{\mathrm{I}}\right\rangle$, where $m_{\mathrm{S}}=\uparrow, \downarrow$ and $m_{\mathrm{I}}= \pm 3 / 2, \pm 1 / 2$. At zero field, the eight eigenstates consist of a triplet (total $\operatorname{spin} F=1$ ) and a quintet $(F=2)$. At $0.65 \mathrm{~T}$, the eight eigenstates are nearly Zeeman product states [see eq. (S3)]. Note that states $|\downarrow,-3 / 2\rangle(|4\rangle)$ and $|\uparrow,+3 / 2\rangle(|8\rangle)$ are exact eigenstates for all values of $B$ field. $\mathbf{d}$, Schematic energy level diagram at $0.65 \mathrm{~T}$ showing ESR (vertical arrows) and NMR (arc arrows) transitions, labeled by I-IV and a-c respectively.

We plot the energy diagram of a $\mathrm{Cu}$ atom in Fig. 2c. The eight eigenstates are labelled as $|i\rangle$ ( $i=1$ to 8). In the limit of high $B$ field (electron Zeeman energy much larger than hyperfine coupling), the eigenstates are nearly the Zeeman product states $\left|m_{\mathrm{S}}, m_{\mathrm{I}}\right\rangle$, where $m_{\mathrm{S}}=\uparrow, \downarrow$ and $m_{\mathrm{I}}= \pm 3 / 2, \pm 1 / 2$. In general, the state $\left|\downarrow, m_{\mathrm{I}}\right\rangle$ hybridizes with $\left|\uparrow, m_{\mathrm{I}}-1\right\rangle\left(m_{\mathrm{I}}=+3 / 2, \pm 1 / 2\right)$ due to the flip-flop hyperfine interaction $\left(S^{+} I^{-}\right.$and $\left.S^{-} I^{+}\right)$, to form three pairs of hybrid states $(|i\rangle$ and $|8-i\rangle$ with $i=$ $1,2,3)^{5}$. For example, at $B=0.65 \mathrm{~T}$, the Zeeman energy is $\sim 6$ times larger than the hyperfine coupling for both isotopes, and states $|\downarrow,+3 / 2\rangle$ and $|\uparrow,+1 / 2\rangle$ mix to form $|1\rangle \approx 0.99|\downarrow,+3 / 2\rangle-0.12|\uparrow,+1 / 2\rangle$ and $|7\rangle \approx 0.12|\downarrow,+3 / 2\rangle+0.99|\uparrow,+1 / 2\rangle$. Due to the slight overlap between eigenstates $|i\rangle$ and $|8-i\rangle$ $\left(\left|\left\langle i\left|S_{z}\right| 8-i\right\rangle\right|^{2} \neq 0\right)$, tunneling electrons can induce state transitions by scattering with the $\mathrm{Cu}$ electron, during which the spin of the tunneling electron is conserved $(\Delta \sigma=0)$. In comparison, $\Delta \sigma=$ \pm 1 transitions (tunneling electron reverses its spin) can occur between $|i\rangle$ and $|9-i\rangle$ since $\left|\left\langle i\left|S_{x}\right| 9-i\right\rangle\right|^{2} \neq 0$. We use these two types of current-induced transitions to control the nuclear polarization as shown below.

The nuclear polarization, $P_{n}=\left\langle I_{\mathrm{z}}\right\rangle / I$, is only $\sim 1.7 \%$ for ${ }^{63} \mathrm{Cu}$ or ${ }^{65} \mathrm{Cu}$ at thermal equilibrium $(B=$ $0.65 \mathrm{~T}, T=1.2 \mathrm{~K}$ ). This polarization results from the Boltzmann occupation of nuclear sub-states that are separated by only $\sim A / 2$. The main thermal relaxation path is likely through scattering by electrons from the Ag substrate 24,32 .

We control the nuclear polarization $P_{n}$ using a spin-polarized current flowing through individual $\mathrm{Cu}$, while the readout of $P_{n}$ is simultaneously realized by taking ESR spectra. Note that the ESRinduced transition rate is much smaller than the current-induced rates (Fig. S5) and thus the ESR process only weakly perturbs $P_{n}$. Figures $3 \mathrm{a}$ and $3 \mathrm{~b}$ show the ESR spectra taken with spin-polarized currents of opposite directions given by opposite bias polarities. The relative amplitudes of the ESR peaks directly reveal the steady-state populations of each nuclear spin state at large $I_{\mathrm{DC}}$. We find that sufficiently large negative bias (electrons tunnel from sample to tip), and thus large current when holding the tip height constant, leads to nuclear spin polarization primarily into the $m_{\mathrm{I}}=-3 / 2$ states (Fig. 3a). Reversing the current direction by applying positive bias leads to a higher occupation of the $m_{\mathrm{I}}=+3 / 2$ states (Fig. $3 \mathrm{~b}$ ). Figure 3 c shows the ESR amplitudes as a function of sample bias $V_{\mathrm{DC}}$ at 
constant tip height. Increasing $V_{\mathrm{DC}}$ and thus the current $I_{\mathrm{DC}}$ increases the degree of nuclear polarization, which saturates to a limiting polarization at large $I_{\mathrm{DC}}$.
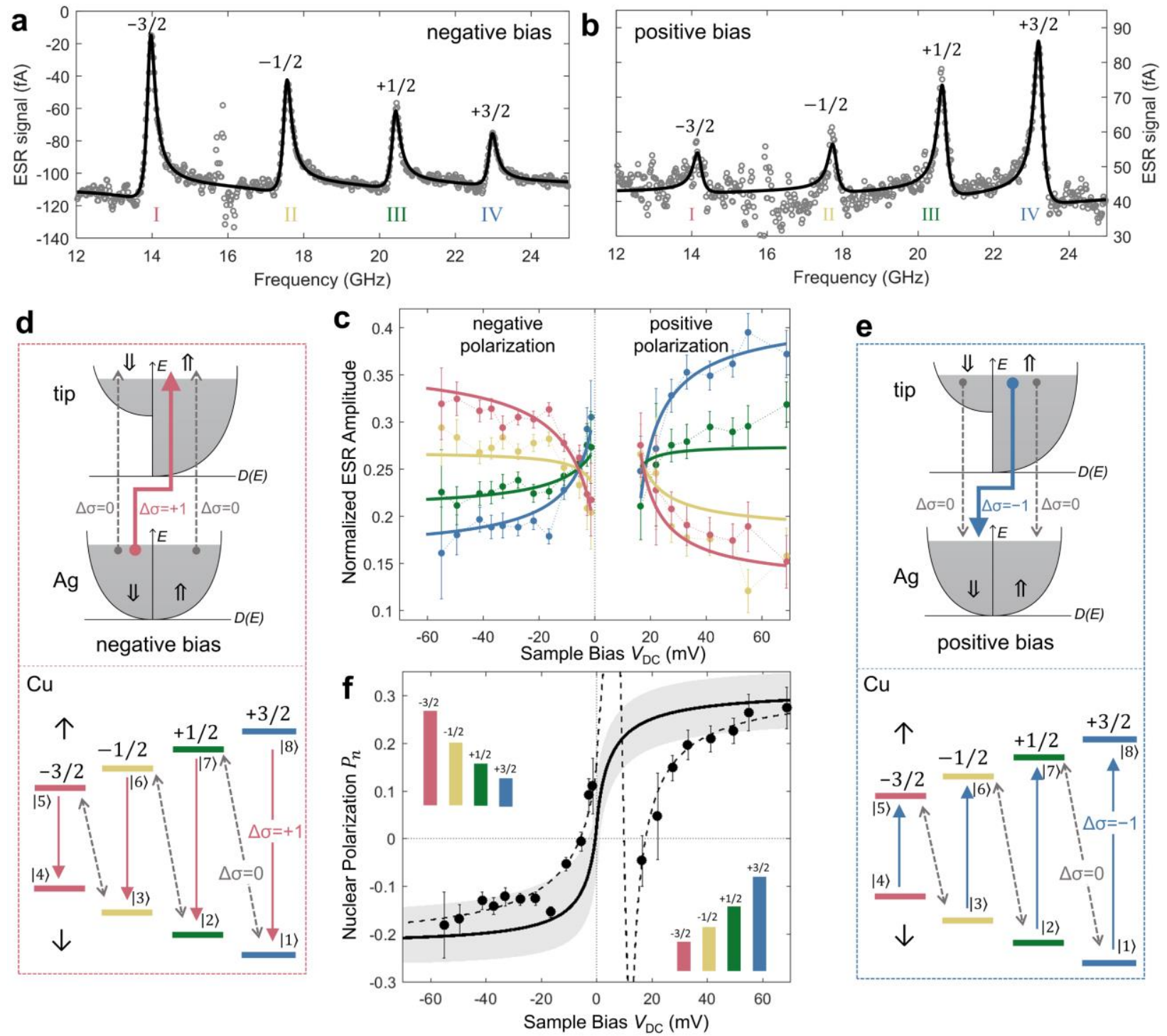

Figure 3 | Spin-transfer torque of the $\mathbf{C u}$ nuclear spin. a and $\mathbf{b}$, ESR spectra of a ${ }^{65} \mathrm{Cu}$ atom at $V_{\mathrm{DC}}=-22 \mathrm{mV}$ and $+22 \mathrm{mV}$ for the same tip $\left(I_{\mathrm{DC}}=60 \mathrm{pA}, V_{\mathrm{RF}}=12 \mathrm{mV}\right.$, total field $B=0.66 \mathrm{~T}$ consisting of an external field of $0.74 \mathrm{~T}$ and an effective tip field, $T=1.2 \mathrm{~K}$ ). c, Normalized ESR amplitudes as a function of sample bias measured with a different tip than in (a, b) (setpoint: $V_{\mathrm{DC}}=22 \mathrm{mV}, I_{\mathrm{DC}}=80 \mathrm{pA} ; V_{\mathrm{RF}}=6-20 \mathrm{mV}, B=0.66 \mathrm{~T}, T=1.2 \mathrm{~K}$ ). Negative and positive sides are fit separately using the rate equation model (see eq. (S15)), giving a tip polarization $\eta$ of $0.14(0.19)$ at negative (positive) bias. d, Mechanism of the nuclear polarization at negative bias $\left(V_{\mathrm{DC}}<0\right)$ ). Upper panel: $\Delta \sigma=+1$ (red arrow) and $\Delta \sigma=0$ (dashed grey arrows) electron tunneling. Lower panel: $\Delta \sigma=+1$ tunneling drives the Cu electron spin from $m_{\mathrm{S}}=\uparrow$ to $\downarrow$ (red arrows); $\Delta \sigma=0$ tunneling drives the flip-flop transitions (dashed grey arrows). The magnetic tip here is anti-aligned with the ground-state $\mathrm{Cu}$ electron spin. Oppositely aligned tip torques the nuclear spin in the opposite directions (see Fig. S3). The weaker tunneling process having opposite spin-flip sign $(\Delta \sigma=-1)$ is also present (not pictured). e, Mechanism of the nuclear polarization at positive bias $\left(V_{\mathrm{DC}}>0\right)$. The weaker tunneling process $(\Delta \sigma=+1)$ is also present (not pictured). f, Nuclear polarization $P_{n}$ as a function of $V_{\mathrm{DC}}$ (solid curve), based on fits to results in (c) using 
the rate equation model. The uncertainty of $P_{n}$ is depicted as the shaded region by considering the uncertainties of the fitting parameters with 95\% confidence. Dashed curve is the asymmetry in ESR peak amplitudes calculated from the same model [see eq. (S17)]. The asymmetry approaches $P_{n}$ in the limit of large bias, but deviates from the true nuclear polarization at small current. Data points are the measured asymmetry obtained directly by weighting the four ESR amplitudes of the same $V_{\mathrm{DC}}$ in (c). The error bars are propagated from the fitting uncertainties of the ESR peak amplitudes at each $V_{D C}$.

The current-controlled nuclear polarization is a consequence of the conservation of total spin angular momentum through two spin-transfer torque ${ }^{33}$ processes at the single-atom level (Fig. 1b). The principle is illustrated in Figs. $3 \mathrm{~d}$ and $3 \mathrm{e}$ for each bias polarity. For example, at positive $V_{\mathrm{DC}}$, tunneling electrons firstly exchange spin angular momentum with the localized $\mathrm{Cu}$ electron spin by exchange scattering (blue arrow in the upper panel of Fig. 3e). This spin-reversing $(\Delta \sigma=-1)$ electron tunneling pumps the electron spin of the $\mathrm{Cu}$ atom from the four lower states to the upper states $\left(\Delta m_{\mathrm{S}}=+1\right)$ to conserve angular momentum ${ }^{32}$. At large $I_{\mathrm{DC}}$, the population ratio between the two states with the same $m_{\mathrm{I}}$ (between $|i\rangle$ and $|9-i\rangle$ ) is set by the spin polarization $(\eta)$ of the tip, to give a ratio of $(1+\eta) /(1-\eta)$, where $\eta \approx 0.19$ for this tip (from the fit in Fig. 3c). The second step of the spin-transfer torque occurs between the nuclear and electron spins of the $\mathrm{Cu}$ atom via the flip-flop hyperfine interaction $\left(S^{+} I^{-}\right.$and $\left.S^{-} I^{+}\right)$. At large $I_{\mathrm{DC}}$, this process is mainly driven by the spinconserving $(\Delta \sigma=0)$ electron tunneling events (dashed grey arrows in Fig. 3e), which equalize the populations of the connected states $(|i\rangle$ and $|8-i\rangle)$. At low $I_{\mathrm{DC}}$, scattering of electrons from the $\mathrm{Ag}$ substrate sets the Boltzmann distribution of the connected states.

Considering all transitions, the state population at positive $V_{\mathrm{DC}}$ follows the paths indicated by the arrows in Fig. 3e, and the net effect is that the nuclear spin is driven towards the states $|1\rangle$ and $|8\rangle$, which are mainly composed of $m_{\mathrm{I}}=+3 / 2$, giving positive nuclear spin polarization $P_{n}$. Similarly, at negative sample bias, the populations are driven to $m_{\mathrm{I}}=-3 / 2$ states (Fig. $3 \mathrm{~d}$ ), giving negative $P_{n}$.

To quantitatively describe the behavior of the electron and nuclear spin of $\mathrm{Cu}$ under the influence of the spin-polarized current, we developed a rate equation model (Supplementary Sec. 6). This model considers transition rates between states due to scattering with electrons that tunnel between tip and Ag, as well as scattering by Ag substrate electrons (originating from Ag and returning to Ag), using a quantum mechanical transition intensity operator ${ }^{32}$. We treat the ESR-induced rates as perturbations (Fig. S5). By fitting the ESR amplitudes versus $V_{\mathrm{DC}}$ in Fig. 3c to our model, we obtained the nuclear polarization $P_{n}$ at different $V_{\mathrm{DC}}$ (Fig. 3f, solid curve). At large current, $P_{n}$ can also be approximated from the ratios of the four ESR amplitudes at each $V_{\mathrm{DC}}$ (see dashed curve in Fig. 3f and Supplementary Sec. 7). The ESR amplitudes (Fig. 3c) along with the corresponding calculated $P_{n}$ (Fig. 3f, dashed curve) show an asymmetry with respect to zero bias, due to different spin pumping directions at opposite bias polarities. At positive $V_{\mathrm{DC}}$, thermal relaxation due to substrate electrons, 
and $\Delta \sigma=-1$ processes due to the spin-polarized tunnel current compete with each other, while at negative $V_{\mathrm{DC}}$ both thermal relaxation and $\Delta \sigma=+1$ processes favor occupation of the lower-energy states.

The model shows that $P_{n}$ grows monotonically with $V_{\mathrm{DC}}$ and the polarization direction is controlled by the bias polarity. The result is a fast electrical initialization of the nuclear spin. We find that when the exchange scattering with tunneling electrons occurs more frequently than the spin relaxation driven by substrate electrons (every $\sim 10 \mathrm{~ns}$ as estimated from the point contact conductance ${ }^{24}$ ), the nuclear spin population starts to deviate from the thermal equilibrium. The saturation polarization at $+70 \mathrm{mV}$ is $\sim 30 \%$, which is a $\sim 17$-fold enhancement over the thermal equilibrium. This corresponds to an effective nuclear spin temperature of $\sim 200 \mathrm{mK}$, which is 6 times cooler than the experimental temperature of $1.2 \mathrm{~K}$. The model also shows that the saturation polarization increases monotonically with the tip spin polarization, and reaches unity with a fully polarized tip ( $\eta=1$ ) (Fig. S4). The time needed to reach the steady-state polarization is limited by the rates of the flip-flop transitions $(\Delta \sigma=0)$, which are slower than the $\Delta \sigma= \pm 1$ transitions by a factor of $\alpha=\left(\gamma_{e} B / A\right)^{2} \approx 40$. The settling time is thus $\sim 4 \alpha \cdot\left(e / I_{\mathrm{DC}}\right)=420 \mathrm{~ns}$ at $I_{\mathrm{DC}}$ of $60 \mathrm{pA}^{32}$.
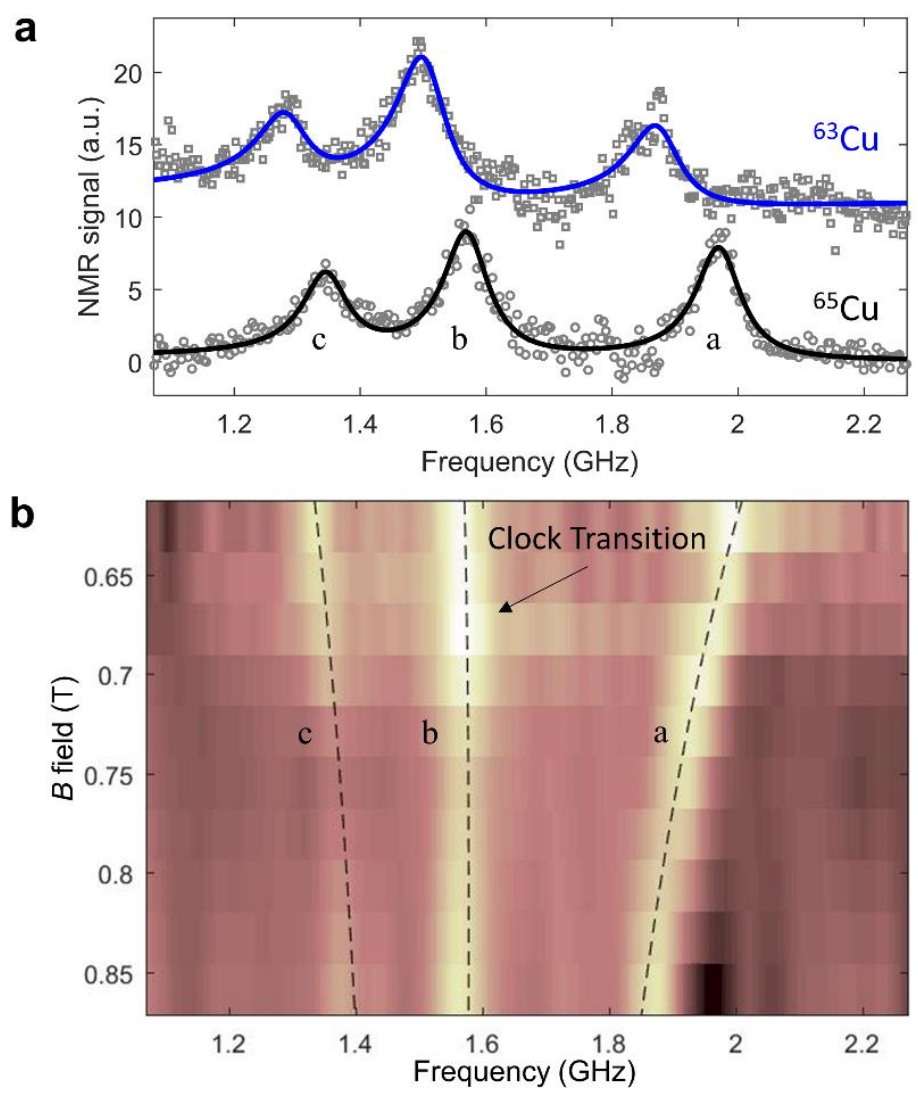

Figure 4 | NMR-type transitions of single $\mathrm{Cu}$ atoms. a, NMR spectra of single ${ }^{63} \mathrm{Cu}$ and ${ }^{65} \mathrm{Cu}$ atoms on $\mathrm{MgO}$ (setpoint: $V_{\mathrm{DC}}=-22 \mathrm{mV}, I_{\mathrm{DC}}=-60 \mathrm{pA} ; V_{\mathrm{RF}}=25 \mathrm{mV}, B=0.66 \mathrm{~T}, T=1.2 \mathrm{~K}$ ). Transitions are labeled as in Fig. $2 \mathrm{~d}$, 
and detected by the change of the magneto-resistance of the electron states using the DC current, different than the traditional double resonance technique (see Fig. $\mathrm{S} 6$ for double resonance spectra) ${ }^{4}$. Transitions between upper four states $\left(m_{\mathrm{S}}=\uparrow\right)$ may also contribute to the spectrum, but these should be much weaker due to their small populations at this spin torque polarity. Solid lines are the fit to three Lorentzian peaks. Spectra are vertically shifted for clarity. b, NMR spectra of a ${ }^{65} \mathrm{Cu}$ atom as a function of total $B$ field (setpoint: $V_{\mathrm{DC}}=16-22$ $\mathrm{mV}, I_{\mathrm{DC}}=60 \mathrm{pA}$ ). The total $B$ field is varied by changing the tip field at an external field of $0.74 \mathrm{~T}$. The values of the total $B$ field are obtained by measuring the ESR spectra of $\mathrm{Cu}$ for each tip position. Dashed lines are the calculated transition frequencies.

The polarized nuclear spin permits direct access to the resonant transitions between adjacent nuclear spin states ${ }^{4,34}$ (changing $m_{\mathrm{I}}$ and leaving $m_{\mathrm{s}}$ unchanged) at the single atom scale (Fig. 4a). These NMR-type transitions ${ }^{35}$ (labeled NMR in the following for brevity) are driven by an AC voltage applied at one of the transition frequencies $(\sim A / 2)$, and are likely made possible by the mixing of electron and nuclear states (Supplementary Sec. 6) ${ }^{5}$. The resonances are detected by the change of electron spin polarization (and thus the magneto-resistance) that results from the NMR transitions. The NMR spectrum of single $\mathrm{Cu}$ atoms reveals three peaks, corresponding to transitions a-c labeled in Fig. 2d. The separation of NMR frequencies makes it possible to individually address each transition. The uneven spacing of nuclear states is due to the hyperfine coupling. The relatively broad NMR peaks compared to other nuclear spin systems ${ }^{6-8}$ are due to a shorter nuclear coherence time, which is limited by the electron spin relaxation time resulting from the interaction with the tunneling current as well as the scattering electrons from the Ag substrate.

We employ the NMR spectra to probe the local magnetic environment of a ${ }^{65} \mathrm{Cu}$ atom by varying the effective tip magnetic field ${ }^{28}$ applied to the atom. The change of the Zeeman energy of the $\mathrm{Cu}$ electron spin manifests as an NMR frequency shift (Fig. 4b). Two frequencies (a and c) shift with the tip magnetic field while the frequency of $b$ is almost constant at $\sim 1.6 \mathrm{GHz}$, which agrees well with the calculated evolution (dashed lines in Fig. 4b). The transition b is an NMR-type clock transition ${ }^{35,36}$, a transition that is insensitive to the $B$ field. The clock transition of ${ }^{63} \mathrm{Cu}$ occurs at a smaller frequency of $\sim 1.5 \mathrm{GHz}$ (Fig. 4a) due to its smaller hyperfine constant. The clock transition frequency can thus be used to distinguish two $\mathrm{Cu}$ isotopes.

The nuclear spin-transfer torque effect shown here should be applicable to other electron-nuclear quantum devices with nuclear spins coupled to unpaired electrons ${ }^{5-8}$. Although the nuclear spin relaxation time of $\mathrm{Cu}$ on $\mathrm{MgO}$ is not yet long enough to perform single-shot readout of nuclear spin states, it should be possible to improve this by using a thicker MgO layers or by using Cu-based molecules ${ }^{37}$. The electrical polarization, driving, and detection of the nuclear spin states provides a new means of local spin manipulation for nuclear spintronics ${ }^{34}$ and detection of the atomic-scale magnetic environment in nanomagnets ${ }^{16,17}$. 


\section{References}

1 T. Staudacher, F. Shi, S. Pezzagna, J. Meijer, J. Du, C. A. Meriles, F. Reinhard \& J. Wrachtrup. Nuclear magnetic resonance spectroscopy on a (5-nanometer) ${ }^{3}$ sample volume. Science 339, 561-563 (2013).

2 F. Shi, Q. Zhang, P. Wang, H. Sun, J. Wang, X. Rong, M. Chen, C. Ju, F. Reinhard, H. Chen, J. Wrachtrup, J. Wang \& J. Du. Single-protein spin resonance spectroscopy under ambient conditions. Science 347, 1135-1138 (2015).

3 M. Fu, T. Imai, T.-H. Han \& Y. S. Lee. Evidence for a gapped spin-liquid ground state in a kagome Heisenberg antiferromagnet. Science 350, 655-658 (2015).

4 C. P. Slichter. Principles of magnetic resonance. (Springer, 1996).

5 G. W. Morley, P. Lueders, M. Hamed Mohammady, S. J. Balian, G. Aeppli, C. W. M. Kay, W. M. Witzel, G. Jeschke \& T. S. Monteiro. Quantum control of hybrid nuclear-electronic qubits. Nat. Mater. 12, 103-107 (2013).

6 S. Thiele, F. Balestro, R. Ballou, S. Klyatskaya, M. Ruben \& W. Wernsdorfer. Electrically driven nuclear spin resonance in single-molecule magnets. Science 344, 1135-1138 (2014).

7 J. J. Pla, K. Y. Tan, J. P. Dehollain, W. H. Lim, J. J. L. Morton, F. A. Zwanenburg, D. N. Jamieson, A. S. Dzurak \& A. Morello. High-fidelity readout and control of a nuclear spin qubit in silicon. Nature 496, 334-338 (2013).

8 A. J. Sigillito, A. M. Tyryshkin, T. Schenkel, A. A. Houck \& S. A. Lyon. All-electric control of donor nuclear spin qubits in silicon. Nat. Nanotechnol. 12, 958-962 (2017).

9 B. Urbaszek, X. Marie, T. Amand, O. Krebs, P. Voisin, P. Maletinsky, A. Högele \& A. Imamoglu. Nuclear spin physics in quantum dots: An optical investigation. Rev. Mod. Phys. 85, 79-133 (2013).

10 D. R. McCamey, J. van Tol, G. W. Morley \& C. Boehme. Fast nuclear spin hyperpolarization of phosphorus in silicon. Phys. Rev. Lett. 102, 027601 (2009).

11 J. H. Smet, R. A. Deutschmann, F. Ertl, W. Wegscheider, G. Abstreiter \& K. von Klitzing. Gatevoltage control of spin interactions between electrons and nuclei in a semiconductor. Nature 415, 281 (2002).

12 M. V. G. Dutt, L. Childress, L. Jiang, E. Togan, J. Maze, F. Jelezko, A. S. Zibrov, P. R. Hemmer \& M. D. Lukin. Quantum register based on individual electronic and nuclear spin qubits in diamond. Science 316, 1312-1316 (2007).

13 H. Alloul, T. Ohno \& P. Mendels. ${ }^{9}$ Y NMR evidence for a fermi-liquid behavior in $\mathrm{YBa}_{2} \mathrm{Cu}_{3} \mathrm{O}_{6+\mathrm{x}}$. Phys. Rev. Lett. 63, 1700-1703 (1989). 
14 T. Wu, H. Mayaffre, S. Krämer, M. Horvatić, C. Berthier, W. N. Hardy, R. Liang, D. A. Bonn \& M.-H. Julien. Magnetic-field-induced charge-stripe order in the high-temperature superconductor $\mathrm{YBa}_{2} \mathrm{Cu}_{3} \mathrm{O}_{\mathrm{y}}$. Nature 477, 191 (2011).

15 P. Mendels, A. Keren, L. Limot, M. Mekata, G. Collin \& M. Horvatić. Ga NMR study of the local susceptibility in kagomé-based $\mathrm{SrCr}_{8} \mathrm{Ga}_{4} \mathrm{O}_{19}$ : Pseudogap and paramagnetic defects. Phys. Rev. Lett. 85, 3496-3499 (2000).

16 F. Borsa, A. Lascialfari \& Y. Furukawa. NMR in Magnetic Molecular Rings and Clusters. In: Novel NMR and EPR techniques (pp. 297-349, eds Janez Dolinšek, Marija Vilfan, \& Slobodan Žumer, Springer Berlin Heidelberg, 2006).

17 E. Micotti, Y. Furukawa, K. Kumagai, S. Carretta, A. Lascialfari, F. Borsa, G. A. Timco \& R. E. P. Winpenny. Local spin moment distribution in antiferromagnetic molecular rings probed by NMR. Phys. Rev. Lett. 97, 267204 (2006).

18 A. W. Overhauser. Polarization of nuclei in metals. Phys. Rev. 92, 411-415 (1953).

19 M. C. Cassidy, H. R. Chan, B. D. Ross, P. K. Bhattacharya \& C. M. Marcus. In vivo magnetic resonance imaging of hyperpolarized silicon particles. Nat. Nanotechnol. 8, 363 (2013).

20 S. Simmons, R. M. Brown, H. Riemann, N. V. Abrosimov, P. Becker, H.-J. Pohl, M. L. W. Thewalt, K. M. Itoh \& J. J. L. Morton. Entanglement in a solid-state spin ensemble. Nature 470, 69 (2011).

21 G. W. Morley, M. Warner, A. M. Stoneham, P. T. Greenland, J. van Tol, C. W. M. Kay \& G. Aeppli. The initialization and manipulation of quantum information stored in silicon by bismuth dopants. Nat. Mater. 9, 725 (2010).

22 C. C. Lo, C. D. Weis, J. van Tol, J. Bokor \& T. Schenkel. All-electrical nuclear spin polarization of donors in silicon. Phys. Rev. Lett. 110, 057601 (2013).

23 C. J. Trowbridge, B. M. Norman, Y. K. Kato, D. D. Awschalom \& V. Sih. Dynamic nuclear polarization from current-induced electron spin polarization. Phys. Rev. B 90, 085122 (2014).

24 W. Paul, K. Yang, S. Baumann, N. Romming, T. Choi, C. P. Lutz \& A. J. Heinrich. Control of the millisecond spin lifetime of an electrically probed atom. Nat. Phys. 13, 403-407 (2017).

25 Y. Ting \& H. Lew. Hyperfine structure of $\mathrm{Cu}^{63}$ and $\mathrm{Cu}^{65}$. Phys. Rev. 105, 581-588 (1957).

26 A. J. Heinrich, J. A. Gupta, C. P. Lutz \& D. M. Eigler. Single-atom spin-flip spectroscopy. Science 306, 466-469 (2004).

27 S. Baumann, W. Paul, T. Choi, C. P. Lutz, A. Ardavan \& A. J. Heinrich. Electron paramagnetic resonance of individual atoms on a surface. Science 350, 417-420 (2015). 
28 K. Yang, Y. Bae, W. Paul, F. D. Natterer, P. Willke, J. L. Lado, A. Ferrón, T. Choi, J. FernándezRossier, A. J. Heinrich \& C. P. Lutz. Engineering the eigenstates of coupled spin-1/2 atoms on a surface. Phys. Rev. Lett. 119, 227206 (2017).

29 P. Willke, Y. Bae, K. Yang, J. L. Lado, A. Ferrón, T. Choi, A. Ardavan, J. Fernández-Rossier, A. J. Heinrich \& C. P. Lutz. Hyperfine interaction of individual atoms on a surface. Submitted (2018).

30 A. F. Otte, M. Ternes, K. von Bergmann, S. Loth, H. Brune, C. P. Lutz, C. F. Hirjibehedin \& A. J. Heinrich. The role of magnetic anisotropy in the Kondo effect. Nat. Phys. 4, 847-850 (2008).

31 B. R. McGarvey. The isotropic hyperfine interaction. J. Phys. Chem. 71, 51-66 (1967).

32 S. Loth, K. von Bergmann, M. Ternes, A. F. Otte, C. P. Lutz \& A. J. Heinrich. Controlling the state of quantum spins with electric currents. Nat. Phys. 6, 340-344 (2010).

33 A. Brataas, A. D. Kent \& H. Ohno. Current-induced torques in magnetic materials. Nat. Mater. 11, 372 (2012).

34 J. A. Reimer. Nuclear hyperpolarization in solids and the prospects for nuclear spintronics. Solid State Nucl. Magn. Reson. 37, 3-12 (2010).

35 G. Wolfowicz, A. M. Tyryshkin, R. E. George, H. Riemann, N. V. Abrosimov, P. Becker, H.-J. Pohl, M. L. W. Thewalt, S. A. Lyon \& J. J. L. Morton. Atomic clock transitions in silicon-based spin qubits. Nat. Nanotechnol. 8, 561-564 (2013).

36 M. Shiddiq, D. Komijani, Y. Duan, A. Gaita-Ariño, E. Coronado \& S. Hill. Enhancing coherence in molecular spin qubits via atomic clock transitions. Nature 531, 348-351 (2016).

37 B. W. Heinrich, L. Braun, J. I. Pascual \& K. J. Franke. Protection of excited spin states by a superconducting energy gap. Nat. Phys. 9, 765 (2013). 


\section{Acknowledgements}

We thank Bruce Melior for expert technical assistance. We gratefully acknowledge financial support from the Office of Naval Research. P.W., Y.B., T.C. and A.J.H acknowledge support from IBS-R027-D1. P.W. acknowledges support from the Alexander von Humboldt Foundation. A.F. acknowledges CONICET (PIP11220150100327 and PUE-22920170100089CO). J.L.L. thanks financial support from ETH Fellowship program. J.F-R. thanks FCT, under the project "PTDC/FIS-NAN/4662/2014".

\section{Author contributions}

K.Y. and C.P.L. designed the experiment. K.Y., P.W., and Y.B. carried out the STM measurements. K.Y. and C.P.L. performed the analysis and developed the rate equation model. A.F., J.L.L. and J.F-R. performed the DFT calculations. All authors discussed the results and edited the manuscript.

\section{Data availability}

The data that support the plots within this paper and other findings of this study are available from the corresponding author upon reasonable request. 